\title{
SUB-PICOSECOND OPTICAL PULSES AT THE SLS STORAGE RING
}

\author{
G. Ingold, A. Streun, B. Singh, R. Abela, P. Beaud, G. Knopp, L. Rivkin, V. Schlott, Th. Schmidt, \\ H. Sigg, J.F. van der Veen, A. Wrulich, Paul Scherrer Institut, Villigen, Switzerland \\ S. Khan, BESSY, Berlin, Germany
}

\begin{abstract}
We report on the feasibility and expected performance of a sub-picosecond X-ray source at the Swiss Light Source (SLS), based on the electron-beam slicing method. This method has recently been demonstrated at the Advanced Light Source (ALS), using a bending magnet to produce ultrafast optical pulses. To improve the flux of such a source for user experiments, we have studied its realization both in the hard and soft X-ray regime, employing small gap insertion devices (IDs) installed either in one or in two successive straight sections. The most favourable geometry is one in which the modulator and the radiator are positioned in the same straight section of the storage ring.
\end{abstract}

\section{INTRODUCTION}

The beam slicing method [1] employs a femtosecond laser to modulate the energy of an electron bunch over the distance of the laser pulse length. To achieve a sufficient separation of the sliced electrons, the energy modulation must be several times larger than the energy spread of the electron beam $\left(\Delta E \geq 5 \sigma_{E} \simeq 13 \mathrm{MeV}\right.$ at the SLS). Therefore, using state-of-the-art lasers, the electron beam energy cannot be much higher than $2.5 \mathrm{GeV}$.

At the SLS [2] (2.4 GeV) undulators will cover the photon energy range $0.01-18 \mathrm{keV}$. A short period hybrid wiggler is used for energies up to $40 \mathrm{keV}$. The notable features of the ID-based photon sources at the SLS are [3]:

- For soft X-rays: the use of elliptical twin-undulators with variable linear and circular polarisation, allowing oppositely polarized, rapidly switched $(0.01-1 \mathrm{kHz})$ beams under otherwise identical conditions.

- For hard X-rays: the use of small gap, short period undulators to extend the high brightness synchrotron radiation (SR) to $\simeq 18 \mathrm{keV}$ (i.e. into the domain of high energy SR facilities), operating on higher (11th/13th) harmonics.

For intermediate energy storage rings such as the SLS, this opens a unique avenue for time-resolved experiments:

- For the production of short photon pulses, the combined effect of the electron beam slicing method and the use of higher undulator harmonics means that high brightness sub-picosecond X-rays up to $18 \mathrm{keV}$ are accessible at undulator beamlines specially developed at user facilities with a beam energy of about $2.5 \mathrm{GeV}$.

\section{LATTICE MODIFICATIONS}

At the PX-beamline 6S we recently have installed the short period $(24 \mathrm{~mm})$ in-vacuum undulator U24. As the (radiator) source for sub-picosecond X-rays, we studied to install small gap (4-6 mm) undulators of this type also in the long straight section $5 \mathrm{~L}(10.8 \mathrm{~m})$. For this purpose, to lower the vertical $\beta$-function from nominally $3 \mathrm{~m}$ to $\leq 1 \mathrm{~m}$ at the center of the radiator, the optics in the straight section $5 \mathrm{~L}$ needs to be modified.

An additional quadrupole triplet mounted asymmetrically splits the long straight 5L into two short straights of different length with $\beta=1.6 \mathrm{~m}$ and $\beta=0.6 \mathrm{~m}$ respectively at the center (Figure 2). The change of the ring optics is confined between sextupole pairs in the arcs adjacent to the straight 5L. Therefore no optical function at any sextupole will be affected. The betatron phase advance introduced by the modification is $\Delta \Phi_{x}=0$ horizontally and $\Delta \Phi_{y}=\pi$ vertically (" $\pi$-trick"). Since in a regular (non-skew) lattice all normal multipoles contain only even powers of the vertical coordinate $y$, the non-linear beam dynamics remains basically unchanged [4]. Marginal deteriorations are due to a slight increase of the integrated sextupole strength and due to a deviation of $\Delta \Phi_{y}$ from $\pi$ for off-momentum particles $(|\Delta \mathrm{p} / \mathrm{p}| \geq 6 \%)$.

The locally modified optics allows the installation of two small gap insertion devices, either two undulators ('Two-Straight Scenario') or one wiggler and one undulator ('One-Straight Scenario'). A sizeable dispersion function $(\eta)$ is necessary to achieve a transverse spatial separation $(5 \sigma)$ of the energy-modulated electron slice at the radiator. Because of the small vertical beam size, a vertical $\eta$-function will be implemented.

\subsection{Two-Straight Scenario}

Two undulators (U24, U17) in section 5L cover the photon range 2-18 keV. Laser modulation of the electron beam occurs in the modulator (revolver-type wiggler W61/W90) installed in section $4 \mathrm{~S}$, immediately upstream from the radiator straight 5L. Four skew quadrupoles in the arcs adjacent to $4 \mathrm{~S}$ are used to generate a vertical asymmetric $\eta$ bump by coupling the horizontal $\eta$ in the arc to the vertical plane at the location of the modulator [4]. An effective dispersion $\eta_{e f f}=8.5 \mathrm{~mm}$ and $\eta_{e f f}^{\prime} \simeq 0$ at the radiator is generated by a vertical betatron oscillation of the energy modulated electrons induced by the skew quadrupoles. The principle is explained in Figure 1. For electrons with an energy modulation $\geq 0.5 \%$, a vertical spatial separation of 43 $\mu \mathrm{m}$ is achieved. The vertical beam size is $8 \mu \mathrm{m}$. To keep the emittance coupling low $(\simeq 0.5 \%)$, various options for creating the asymmetric $\eta$-bump are under study. 


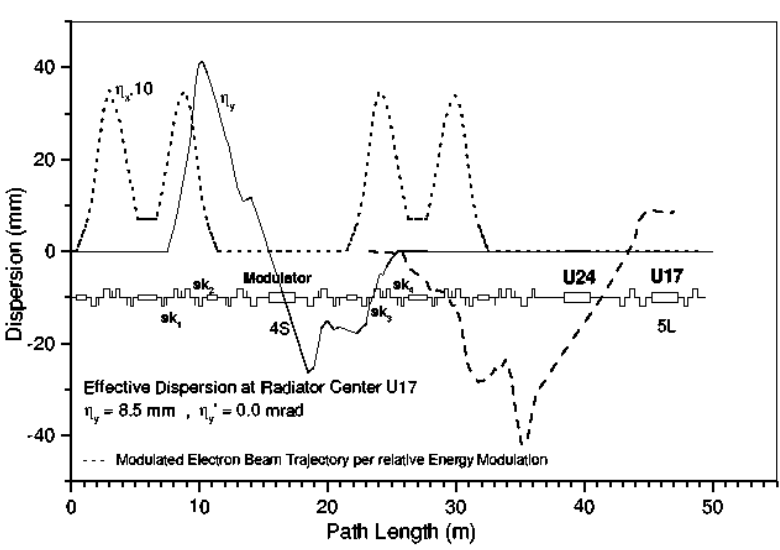

Figure 1: The modulator (in $4 \mathrm{~S}$, with asymmetric $\eta$-bump) and the radiator(s) (in $5 \mathrm{~L}$, with mini-beta lattice) are installed in two successive straight sections. Solid curve: closed vertical dispersion bump of the stored beam. Dashed curve: vertical dispersion of the sliced beam.

\subsection{One-Straight Scenario}

The first radiator (undulator U24) in Figure 1 is replaced by a small gap wiggler as the modulator. The modulator and the radiator are now installed in the same straight section. This scheme, shown in Figure 2, provides the smallest temporal stretching of the electron bunch slice (due to the non-isochronicity of the SLS storage ring) and thus the shortest X-ray pulses. In addition, the modulator wiggler (W110) is now resonant to the laser fundamental $(800 \mathrm{~nm})$, since a simultaneous operation with a second beamline is not required. The closed vertical $\eta$-bump around the modulator is generated by a vertical 4-dipole chicane, providing $\eta=8.8 \mathrm{~mm}$ and $\eta^{\prime}=0$ for the $5 \sigma$-separation $(48 \mu \mathrm{m})$ of the short photon pulses. The vertical displacement $(\simeq 8 \mathrm{~mm})$ of the stored beam in the modulator W110 with respect to the radiator $\mathrm{U} 17$ is used to separate and block the unwanted modulator radiation. Minimizing the photon background is critical for creating a clean image of the source (see section $3.2)$.

\section{SUB-PICOSECOND X-RAY SOURCE}

The laser slicing efficiency is determined by the number of electrons per bunch with an energy modulation $\Delta E \geq$ $0.5 \%$ after the laser interaction in the modulator. By numerical integration we calculate $\Delta E=-e \int \vec{v}_{\perp} \cdot \vec{E}(\vec{x}, t) d t$ where $\vec{v}_{\perp}$ is the electron transverse velocity and $\vec{E}(\vec{x}, t)$ the electrical field of the laser. The waist of the laser beam at the center of the modulator is typically $200 \mu \mathrm{m}(\mathrm{H}) \times 200$ $\mu \mathrm{m}(\mathrm{V})$.

\subsection{Laser, Modulator and Radiator}

The following parameters are used to predict the characteristics of the sub-picosecond X-ray source:

- One-straight scenario $(5 \mathrm{~L})$ :

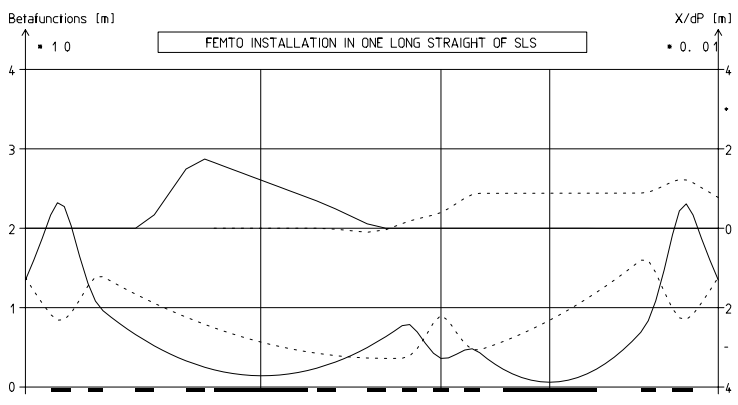

Figure 2: Mini-beta lattice with chicane for pure spatial beam separation. The modulator $\mathrm{W} 110$ and the radiator U17 are installed in the long straight section 5L. Solid curves: vertical beta function and vertical dispersion of the stored beam. Dotted curves: horizontal beta function and vertical dispersion of the sliced beam.

Ti:sapphire laser: $800 \mathrm{~nm}$ (fundamental), $50 \mathrm{fs}$ [FWHM], $1 \mathrm{~mJ} /$ pulse, $5 \mathrm{~W}, 5 \mathrm{kHz}(10 \mathrm{kHz})$; modulator W110: period $110 \mathrm{~mm}$, gap $7.5 \mathrm{~mm}$, peak field $2.5 \mathrm{~T}$, K-parameter 25.6, length $2 \mathrm{~m}$; radiator U17: gap $4 \mathrm{~mm}$, period $17 \mathrm{~mm}$, peak field $1.0 \mathrm{~T}$, energy range $5-18 \mathrm{keV}$ (3.-11. harmonic); slicing efficieny (400 bunches): $5.5 \mathrm{E}-9(5 \mathrm{kHz})$ (the slicing efficiency is defined as the number of electrons with $\Delta E \geq$ $0.5 \%$ / second/ beam current (400 mA)).

- Two-straight scenario (4S / 5L):

Simultaneous operation of the material science (MS) beamline $4 \mathrm{~S}$ requires that the laser is in resonance with the second harmonic of the modulator wiggler. Ti:sapphire laser: $400 \mathrm{~nm}$ (2nd harmonic, conversion efficiency 30-50\%), 50 fs [FWHM], $1 \mathrm{~mJ} /$ pulse, $2 \mathrm{~W}, 2 \mathrm{kHz}(5 \mathrm{kHz})$; modulator W90 (revolver type W61/W90): period $90 \mathrm{~mm}$, gap 7.5 mm, peak field $2.35 \mathrm{~T}$, K-parameter 19.7, length $2 \mathrm{~m}$; (wiggler W61, under construction, for regular operation at the MS-beamline (4S), period $61 \mathrm{~mm}$, gap $7.5 \mathrm{~mm}$, peak field $1.95 \mathrm{~T}$, K-parameter 11.0, length $2 \mathrm{~m}$ ); radiator U24: gap $5 \mathrm{~mm}$, period $24 \mathrm{~mm}$, peak field $1.10 \mathrm{~T}$, energy range 2-8 $\mathrm{keV}$ (1.-7. harmonic); radiator U17: gap 4mm, peak field $1.0 \mathrm{~T}$, energy range 5-18 keV (3.-11. harmonic); slicing efficiency (single bunch): $7.5 \mathrm{E}-7$, slicing efficiency (400 bunches): $4.8 \mathrm{E}-9$ ( $5 \mathrm{kHz})$.

We expect that a $50 \mathrm{fs}$ Ti:sapphire laser system based on the chirped pulse amplification with an average power of 3 $\mathrm{W}(1 \mathrm{~mJ} /$ pulse, $3 \mathrm{kHz})$ will commercially be available at the end of year 2001. Later a $10 \mathrm{~W}$ laser system $(1 \mathrm{~mJ} / \mathrm{pulse}$, $10 \mathrm{kHz}$ ) developed by the Combustion Research Laboratory at PSI will be used.

\subsection{Sub-Picosecond Synchrotron Pulses}

The simulated slicing efficiency $5 \mathrm{E}-9$ for a $5 \mathrm{kHz}$ laser system is in good agreement with the analytical estimate given in [1]. Using this value, the intensity of the subpicosescond X-ray pulses given in Figure 4 is calculated from the values listed in the 3 rd column, which are valid for the regular multibunch operation $(400 \mathrm{~mA}, 500 \mathrm{Mhz})$ 


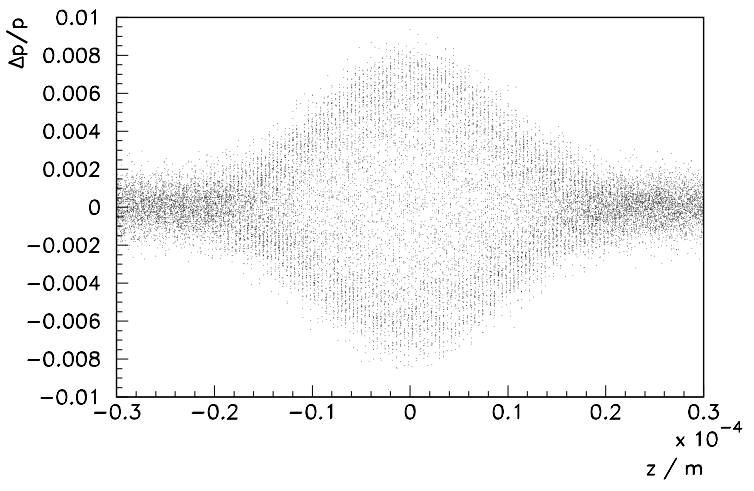

Figure 3: Calculated momentum and spatial distribution of the electron bunch following interaction with the laser in the modulator. An energy modulation $\geq 0.5 \%$ is required.

of the SLS storage ring. The reduction due to the undulator phase error, the emittance (5 nm-rad, coupling $0.3 \%$ ) and the energy spread (9 E-4) of the electron beam, have been taken into account [3].

Imaging will be used to separate the short photon pulses. We follow the concept adopted at the SIM-beamline 11M for time-resolved photoelectron-microscopy on magnetic materials [5] at the SLS (section 3.3). A focussing mirror having demagnification $1 / \mathrm{M}$, located behind the double crystal monochromator ( $\mathrm{Si}(111)$ ), images the two parallelly displaced source points for the sliced and unperturbed electrons in the radiator (see Figure 1,2) onto the intermediate image plane. At the intermediate focus the images are separated by $\Delta^{\prime}=\Delta / M \simeq 25 \mu \mathrm{m}$. A pair of slits, displaced $5 \sigma_{y}$ above the optical axis, will select the sub-picosecond $\mathrm{X}$-ray pulses and block the long-pulse background. A mechanical chopper $(1-5 \mathrm{kHz})$ in front of the slits is used to match the time structure of the X-ray beam to the laser system.

\begin{tabular}{|r|r|c|c|c|}
\hline \multicolumn{5}{|c|}{ Flux: F [photons/sec/0.1\% bw] } \\
\hline \hline $\mathrm{N}$ & $\mathrm{h} \nu$ & $\mathrm{F}$ & $\mathrm{F}$ & $\mathrm{F}$ \\
\hline & {$[\mathrm{keV}]$} & {$[20 \mathrm{ps}, 500 \mathrm{MHz}]$} & {$[20 \mathrm{ps}, \quad 5 \mathrm{kHz}]$} & {$[100 \mathrm{fs}, 5 \mathrm{kHz}]$} \\
\hline \hline 1 & 1.5 & $3.5 \mathrm{E}+15$ & $3.5 \mathrm{E}+10$ & $1.8 \mathrm{E}+7$ \\
\hline 3 & 4.6 & $1.3 \mathrm{E}+15$ & $1.3 \mathrm{E}+10$ & $6.5 \mathrm{E}+6$ \\
\hline 5 & 7.7 & $4.6 \mathrm{E}+14$ & $4.6 \mathrm{E}+9$ & $2.3 \mathrm{E}+6$ \\
\hline 7 & 10.8 & $1.6 \mathrm{E}+14$ & $1.6 \mathrm{E}+9$ & $8.0 \mathrm{E}+5$ \\
\hline 9 & 13.9 & $6.6 \mathrm{E}+13$ & $6.5 \mathrm{E}+8$ & $3.3 \mathrm{E}+5$ \\
\hline 11 & 16.9 & $2.6 \mathrm{E}+13$ & $2.6 \mathrm{E}+8$ & $1.3 \mathrm{E}+5$ \\
\hline 13 & 20.0 & $9.3 \mathrm{E}+12$ & $9.5 \mathrm{E}+7$ & $4.8 \mathrm{E}+4$ \\
\hline
\end{tabular}

\begin{tabular}{|r|r|c|c|c|}
\hline \multicolumn{5}{|c|}{ Brilliance: $\mathbf{B}\left[\mathbf{p h o t o n s} / \mathbf{s e c} / \mathbf{m m}^{2} / \mathbf{m r a d}^{2} / \mathbf{0 . 1 \%} \mathbf{b w}\right]$} \\
\hline \hline $\mathrm{N}$ & $\mathrm{h} \nu$ & $\mathrm{B}$ & $\mathrm{B}$ & $\mathrm{B}$ \\
\hline & {$[\mathrm{keV}]$} & {$[20 \mathrm{ps}, 500 \mathrm{MHz}]$} & {$[20 \mathrm{ps}, \quad 5 \mathrm{kHz}]$} & {$[100 \mathrm{fs}, \quad 5 \mathrm{kHz}]$} \\
\hline \hline 1 & 1.5 & $7.1 \mathrm{E}+19$ & $7.0 \mathrm{E}+14$ & $3.5 \mathrm{E}+11$ \\
\hline 3 & 4.6 & $3.6 \mathrm{E}+19$ & $3.6 \mathrm{E}+14$ & $1.8 \mathrm{E}+11$ \\
\hline 5 & 7.7 & $1.4 \mathrm{E}+19$ & $1.4 \mathrm{E}+14$ & $7.0 \mathrm{E}+10$ \\
\hline 7 & 10.8 & $5.1 \mathrm{E}+18$ & $5.0 \mathrm{E}+13$ & $2.5 \mathrm{E}+10$ \\
\hline 9 & 13.9 & $1.8 \mathrm{E}+18$ & $1.8 \mathrm{E}+13$ & $9.0 \mathrm{E}+9$ \\
\hline 11 & 16.9 & $6.7 \mathrm{E}+17$ & $6.5 \mathrm{E}+12$ & $3.3 \mathrm{E}+9$ \\
\hline 13 & 20.0 & $2.4 \mathrm{E}+17$ & $2.4 \mathrm{E}+12$ & $1.2 \mathrm{E}+9$ \\
\hline
\end{tabular}

Figure 4: Average flux and brightness of the subpicosecond X-ray pulses for the U17 radiator (integrated over $0.25 \mathrm{mrad}(\mathrm{H})$ x $0.05 \mathrm{mrad}(\mathrm{V}))$.

\subsection{Picosecond Synchrotron Pulses}

We also studied the two-straight scenario for the straight sections $10 \mathrm{~S}$ and $11 \mathrm{M}$, where the elliptically polarizing twin-undulators UE56 for beamline 11M would be used as radiators. A similar scheme as shown in Figure 1 would again be applicable. However, time scales of interest for magnetic studies are rather in the pico- and nanosecond range, where instead of slicing the intrinsic time structure of the storage ring ( $20 \mathrm{ps}(\mathrm{rms})$ pulses spaced by $2-800 \mathrm{~ns}$ ) will be used.

The novel feature of beamline $11 \mathrm{M}$ is the concept used for helicity switching. A chicane with 7 dipoles creates a small $( \pm 0.5 \mathrm{~mm})$ parallel displacement of the electron beam in the horizontal direction which is opposite for both undulators. The optical paths for the two photon beams with opposite circular or linear polarization overlap almost completely. A single set of relatively small mirrors is used to accept and guide both photon beams. By imaging the two source points with a demagnifying (4:1) optics, both photon beams are again separated at the intermediate focus. At this location a mechanical chopper alternatively blocks one of the two photon beams. The two photon beams can be overlapped on the sample using a single refocussing mirror.

\section{SUMMARY}

We propose to develop at the SLS a facility for subpicosecond X-ray pulses based on electron-beam slicing method with the characteristics of U17 as the radiator. The undulator will likely be the one planned for the micro$\mathrm{XAFS} /$ diffraction beamline. The facility will enable timedependent studies of the structural dynamics of condensed matter and will complement time resolved studies in the pico- and nanosecond range planned at the photoelectronmicroscopy beamline for soft $\mathrm{X}$-rays.

\section{REFERENCES}

[1] R.W. Schoenlein, S. Chattopadhyay, H.H. Chong, T.E. Glover, P.A. Heimann, C.V. Shank, A.A. Zholents, M.S. Zolotorev, "Generation of Femtosecond Pulses of Synchrotron Radiation", SCIENCE 287 (2000) 2237 and Appl. Phys. B71 (2000) 1.

[2] A. Streun for the SLS team, "Commissioning of the Swiss Light Source", these proceedings.

[3] G. Ingold, "Insertion Devices: New Concepts and Performance", Proceedings of EPAC 2000, Vienna, Austria (2000), 220.

[4] Y. Wu, H. Nishimura, D.S. Robin, A.A. Zholents, E. Forest, "Mini-Beta Lattice for the Femto-Second X-Ray Source at the ALS", Proceedings of EPAC 2000, Vienna, Austria (2000), 1098.

[5] C. Quitmann, U. Flechsig, L. Patthey, Th. Schmidt, G. Ingold, M. Howells, M. Janousch, R. Abela, "A Beamline for Time Resolved Photoelectron-Microscopy on Magnetic Materials at the Swiss Light Source (SLS)", Proc. of 2nd LEEM/PEEM Workshop, Paris, Sept. 2000, "Surface Science". 